\title{
Synthesis of new calix[4]arenes bearing silylether groups
}

\author{
Kazem D. Safa* and Yones Mosaei Oskoei \\ Organosilicon Research Laboratory, Faculty of Chemistry, University of Tabriz, 5166616471 \\ Tabriz, Iran \\ E-mail: dsafa@tabrizu.ac.ir
}

\begin{abstract}
The Si-H groups of 25,26,27,28-tetrakis[4-(tris(dimethylsilyl)methyl)butoxy]calix[4]arene (II) were treated with methanol, ethanol, propanol, butanol, pentanol, hexanol, isopropanol, 1-methyl propanol, 2-methylpropanol and 2-chloroethanol in the presence of Karstedt catalyst (platinum(0)-1,3-divinyl-1,1,3,3-tetramethyldisiloxane complex, solution in xylene) to give the corresponding 25,26,27,28-tetrakis[4-(tris(alkoxydimethylsilyl)methyl)butoxy]calix[4]arene. It is found that alcoholysis of calix[4] arene 1 in the presence of Speier catalyst $\left(\mathrm{H}_{2} \mathrm{PtCl}_{6} \cdot 6 \mathrm{H}_{2} \mathrm{O}\right)$ was unsuccessful using reflux conditions over seven days. In addition, the rate of alcoholysis is dependent on the amounts of the catalyst and reaction temperature.
\end{abstract}

Keywords: Calixarene, sterically-hindered groups, organosilicon, alcoholysis, silyl ethers

\section{Introduction}

Calixarenes ${ }^{1}$ have been widely studied as hosts and potential hosts for molecular recognition. Calixarenes containing organosilicon groups are of potential interest for molecular recognition of anions and nucleophilic substances, ${ }^{2-4}$ as many silicon compounds can interact with nucleophiles to form hypervalent silicon adducts. ${ }^{5}$ To date, only a few calixarenes containing organosilicon groups have been reported. ${ }^{6-11}$

Among the diverse reactions at silicon centers, those involving silicon - oxygen bond formation are particularly important and silyl ethers are among the most widely used protecting groups for hydroxyl functions in organic synthesis, ${ }^{12}$ and also for the preparation of "prodrugs" for drug delivery systems. ${ }^{13}$ They also play an important role in inorganic synthesis as precursors in the preparation of sol-gels and other condensed siloxane materials. ${ }^{14}$ The dehydrocoupling reaction between hydrosilane and an alcohol is a typical well-known method for the preparation of Si-O bonds using transition-metal catalysts. ${ }^{15}$ We have recently developed a convenient procedure for the preparation of a series of tris(alkoxydimethylsilyl)methanes by the reaction of $\left(\mathrm{HMe}_{2} \mathrm{Si}\right)_{3} \mathrm{CH}$ with monofunctional alcohols in the presence of chloroplatinic acid 
$\left(\mathrm{H}_{2} \mathrm{PtCl}_{6} \cdot 6 \mathrm{H}_{2} \mathrm{O}\right)$ as catalyst. ${ }^{16}$ Since alkoxysilane-calixarenes represent a new class of calixarenes, our interest in the preparation of calix[4]arenes bearing the tris(alkoxydimethylsilyl)methyl group was greatly enhanced. Herein we report the use of the dehydrocoupling reaction for the preparation of calixarenes containing silyl ether groups via reaction between a calixarene bearing $\mathrm{Si}-\mathrm{H}$ group and various alcohols in the presence of Karstedt catalyst.

\section{Results and Discussion}

$\left(\mathrm{HMe}_{2} \mathrm{Si}\right)_{3} \mathrm{CH}$ was prepared by the reaction of $\mathrm{CHBr}_{3}$ and $\mathrm{Mg}$ with $\mathrm{HMe}_{2} \mathrm{SiCl}$ in THF. ${ }^{17,18}$ The solvated organolithium reagent $\left(\mathrm{HMe}_{2} \mathrm{Si}\right)_{3} \mathrm{CLi}$, was obtained by treatment of $\left(\mathrm{HMe}_{2} \mathrm{Si}\right)_{3} \mathrm{CH}$ with lithium diisopropyl amide (LDA) at room temperature. ${ }^{19}$ 25,26,27,28-Tetrakis(4-bromobutoxy) calix[4]arene 1 was synthesized by treatment of the phenol containing calixarene with excess $\mathrm{NaH}$ and 1,4-dibromobutane in DMF. The desired calixarene precursor bearing the $\left(\mathrm{HMe}_{2} \mathrm{Si}\right)_{3} \mathrm{C}$ groups was obtained by the reaction of $\left(\mathrm{HMe}_{2} \mathrm{Si}\right)_{3} \mathrm{CLi}$ with 1 in THF (Scheme 1).

$$
\mathrm{HMe}_{2} \mathrm{SiCl}+\mathrm{CHBr}_{3}+\mathrm{Mg} \stackrel{\mathrm{THF}}{\longrightarrow}\left(\mathrm{HSiMe}_{2}\right)_{3} \mathrm{CH} \stackrel{\mathrm{LDA}}{12 \mathrm{~h}}\left(\mathrm{HSiMe}_{2}\right)_{3} \mathrm{CLi}
$$

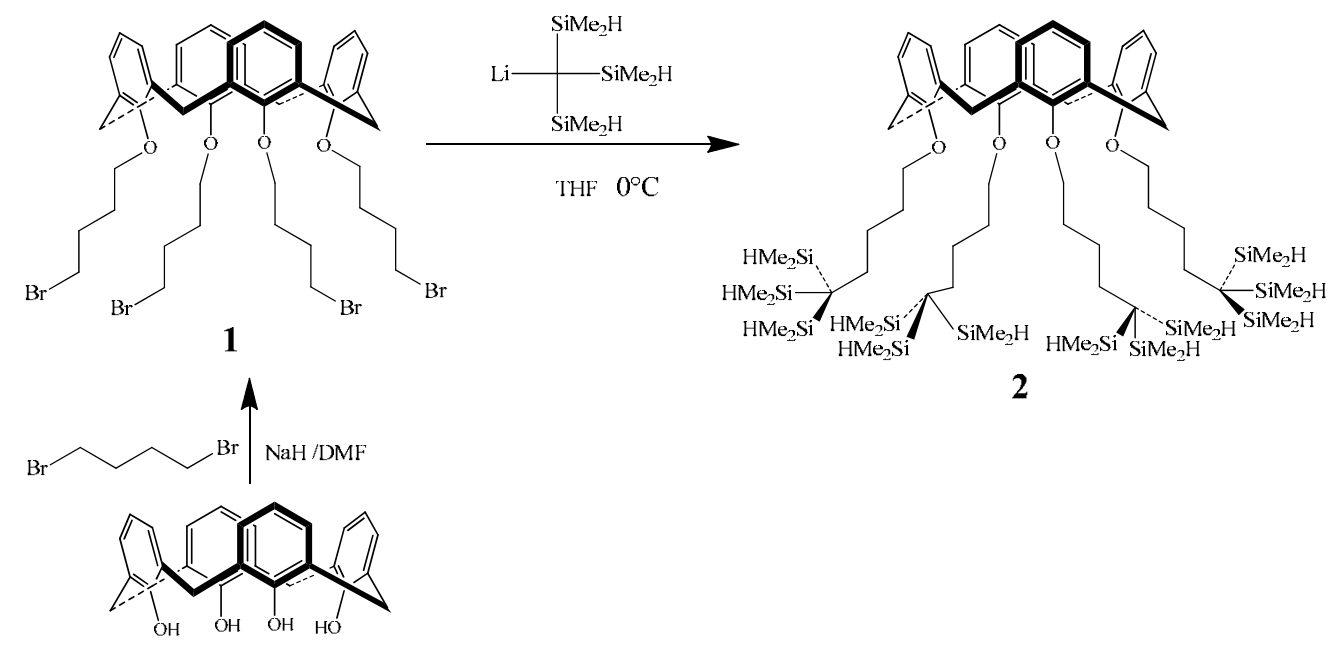

Scheme 1. Preparation of 25,26,27,28-tetrakis[4-(tris(dimethylsilyl)methyl)butoxy]calix[4]arene 2.

We have recently reported the preparation of tris(alkoxydimethylsilyl)methanes via the reaction of tris(dimethylsilyl)methane and various alcohols in the presence of the Speier catalyst $\left(\mathrm{H}_{2} \mathrm{PtCl}_{6} \cdot 6 \mathrm{H}_{2} \mathrm{O}\right)$ under mild and aerobic conditions ${ }^{16}$ (Scheme 2). Since we were interested in extending this methodology to the coupling of the calixarene bearing $\mathrm{Si}-\mathrm{H}$ groups such as 2 with 
various alcohols, it was decided to study the dehydrocoupling between compound 2 and some alcohols in different conditions (Scheme 3).

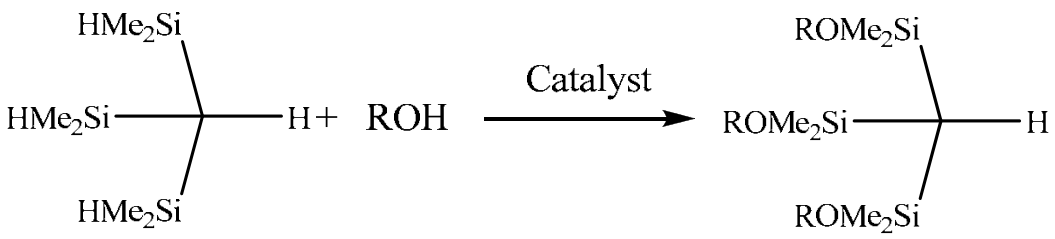

Scheme 2. Preparation of tris(alkoxydimethylsilyl)methanes.

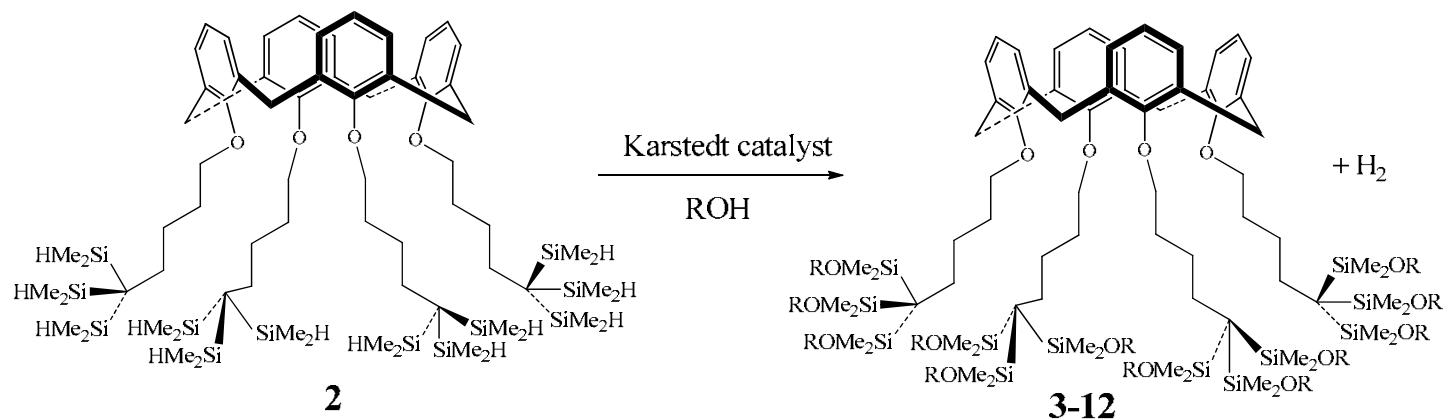

Scheme 3. Preparation of calix[4]arene bearing tris(alkoxydimethylsilyl)methane groups.

Alcoholysis of calixarene 2 with primary alcohols (methanol, ethanol, propanol and butanol) failed to go to completion using the Speier catalyst under reflux conditions over seven days. These reactions were therefore repeated using various amounts of the catalyst $([\mathrm{Pt}] /[\mathrm{Si}-\mathrm{H}]=0.055$ to $[\mathrm{Pt}] /[\mathrm{Si}-\mathrm{H}]=0.39)$. However these conditions also failed to achieve a complete reaction.

The Karstedt catalyst (platinum(0)-1,3-divinyl-1,1,3,3-tetramethyldisiloxane complex, solution in xylene) which is more active than the Speier catalyst $\left(\mathrm{H}_{2} \mathrm{PtCl}_{6} \cdot 6 \mathrm{H}_{2} \mathrm{O}\right)$ was then examined. Reaction conditions were optimized by using propanol as a typical alcohol and the Karstedt catalyst at $80^{\circ} \mathrm{C}$. As shown in Figure 1, increasing the loading of the Karstedt catalyst to a certain extent can affect the reaction time. By increasing the amount of Pt catalyst from $[\mathrm{Pt}] /[\mathrm{Si}-\mathrm{H}]=5.4 \times 10^{-4}$ to $[\mathrm{Pt}] /[\mathrm{Si}-\mathrm{H}]=7.2 \times 10^{-3}$, we were able to reduce the reaction time from $36 \mathrm{~h}$ to $8 \mathrm{~h}$. The colorless reaction mixture gradually turned to homogeneous black-colored liquid, indicating the generation of colloidal $\mathrm{Pt}(0)$ particles.

The method was found to be applicable also to secondary alcohols and leads to selective formation of the corresponding calixarenes bearing alkoxysiloxanes in good yields (Table 1). As shown in Table 1 reaction of calixarene 2 with primary alcohols gave higher yields than the analogous reactions with secondary alcohols, probably due to the increase in steric hinderance. 


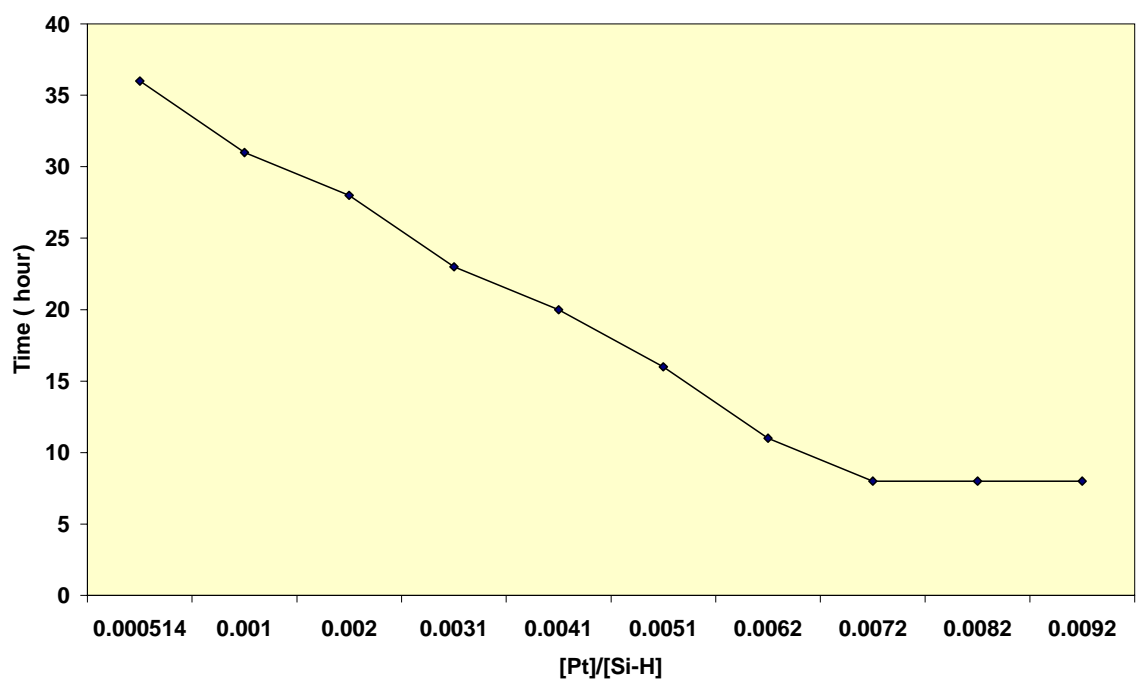

Figure 1. The effect of the amount of the Karstedt catalyst on the reaction time.

Table1. Yields of alcoholysis of calixarene 2 with various alcohols in the presence of the Karstedt catalyst

\begin{tabular}{lll}
\hline Compound & Alcohols & Yields (\%) \\
\hline $\mathbf{3}$ & $\mathrm{CH}_{3} \mathrm{OH}$ & 85 \\
$\mathbf{4}$ & $\mathrm{CH}_{3} \mathrm{CH}_{2} \mathrm{OH}$ & 85 \\
$\mathbf{5}$ & $\mathrm{CH}_{3} \mathrm{CH}_{2} \mathrm{CH}_{2} \mathrm{OH}$ & 83 \\
$\mathbf{6}$ & $\mathrm{CH}_{3} \mathrm{CH}_{2} \mathrm{CH}_{2} \mathrm{CH}_{2} \mathrm{OH}$ & 80 \\
$\mathbf{7}$ & $\mathrm{CH}_{3} \mathrm{CH}_{2} \mathrm{CH}_{2} \mathrm{CH}_{2} \mathrm{CH}_{2} \mathrm{OH}$ & 80 \\
$\mathbf{8}$ & $\mathrm{CH}_{3} \mathrm{CH}_{2} \mathrm{CH}_{2} \mathrm{CH}_{2} \mathrm{CH}_{2} \mathrm{CH}_{2} \mathrm{OH}$ & 80 \\
$\mathbf{9}$ & $\left(\mathrm{CH}_{3}\right)_{2} \mathrm{CHOH}_{3}$ & 77 \\
$\mathbf{1 0}$ & $\mathrm{CH}_{3} \mathrm{CH}_{2}\left(\mathrm{CH}_{3}\right) \mathrm{CHOH}_{2}$ & 75 \\
$\mathbf{1 1}$ & $\mathrm{CH}_{3}\left(\mathrm{CH}_{3}\right) \mathrm{CHCH}$ & $\mathrm{OH}$ \\
$\mathbf{1 2}$ & $\mathrm{ClCH}_{2} \mathrm{CH}_{2} \mathrm{OH}$ & 75 \\
\hline
\end{tabular}

The reaction progress was monitored by FTIR spectroscopy on the basis of absorption measurements at the Si-H stretching bond frequency $\left(2107 \mathrm{~cm}^{-1}\right)$ with reference to the standard curve. The FTIR spectrum of the propoxysilane-bearing calixarene $\mathbf{5}$ does not show a sharp peak at $2107 \mathrm{~cm}^{-1}$ indicating the absence of a Si-H bond, and shows the concomitant appearance of the Si-O peak at $1084 \mathrm{~cm}^{-1}$ (Figure 2). In addition, the ${ }^{1} \mathrm{H}$ NMR spectrum of (5) for example, shows the presence of 36 protons $\left(\mathrm{C}_{3} \mathrm{CH}_{2} \mathrm{CH}_{2} \mathrm{O}\right)$ at $1.1 \mathrm{ppm}$ and 32 protons $\left(12 \times \mathrm{CH}_{3} \mathrm{CH}_{2} \mathrm{C}_{2} \mathrm{O}\right.$, $4 \times \mathrm{CCH}_{2} \mathrm{CH}_{2} \mathrm{CH}_{2} \mathrm{CH}_{2} \mathrm{O}$ ) at 3.5-3.8 ppm (Figure 3). Similar results were observed for other alcohols. 


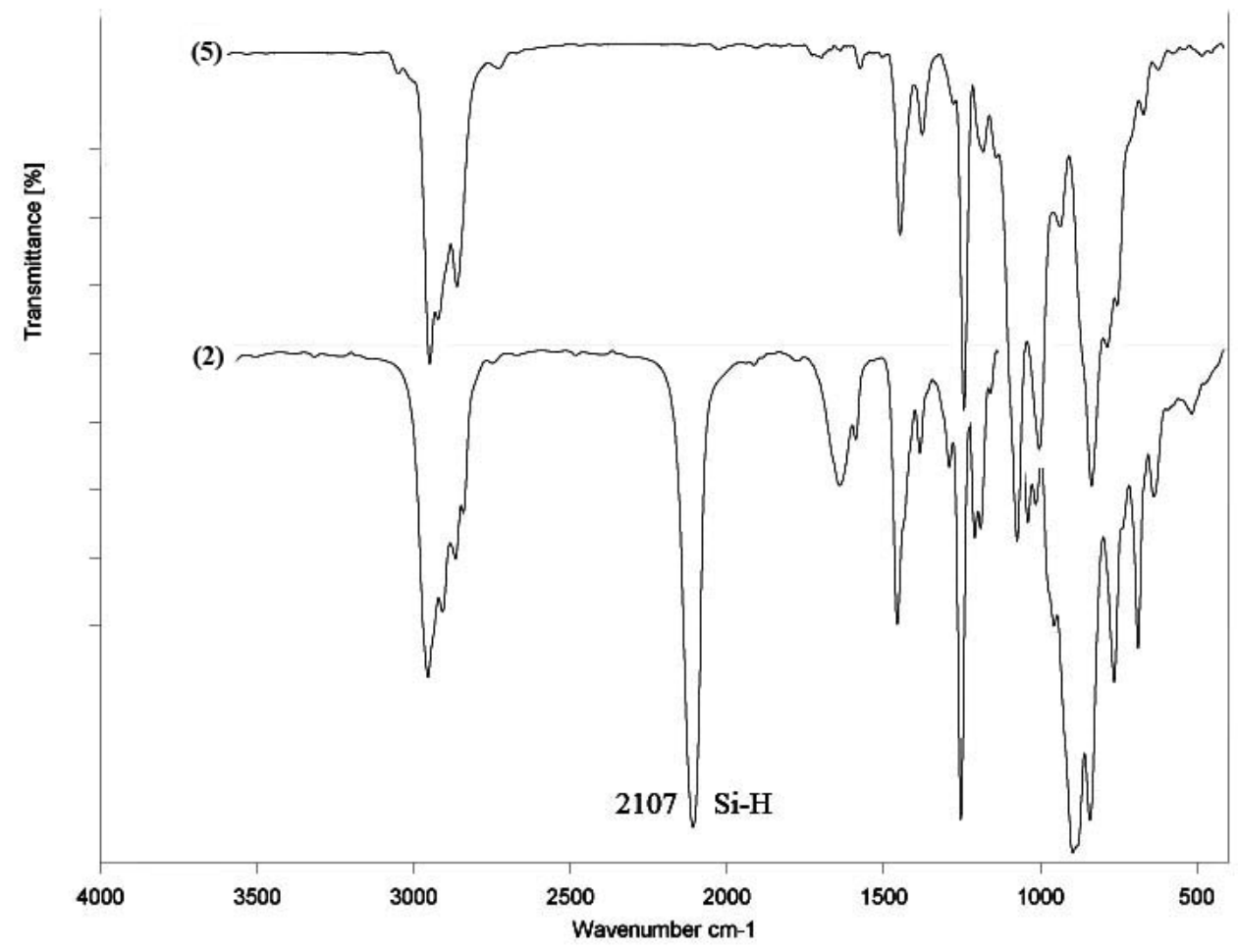

Figure 2. The FTIR spectra of the compounds $\mathbf{2}$ and $\mathbf{5}$.

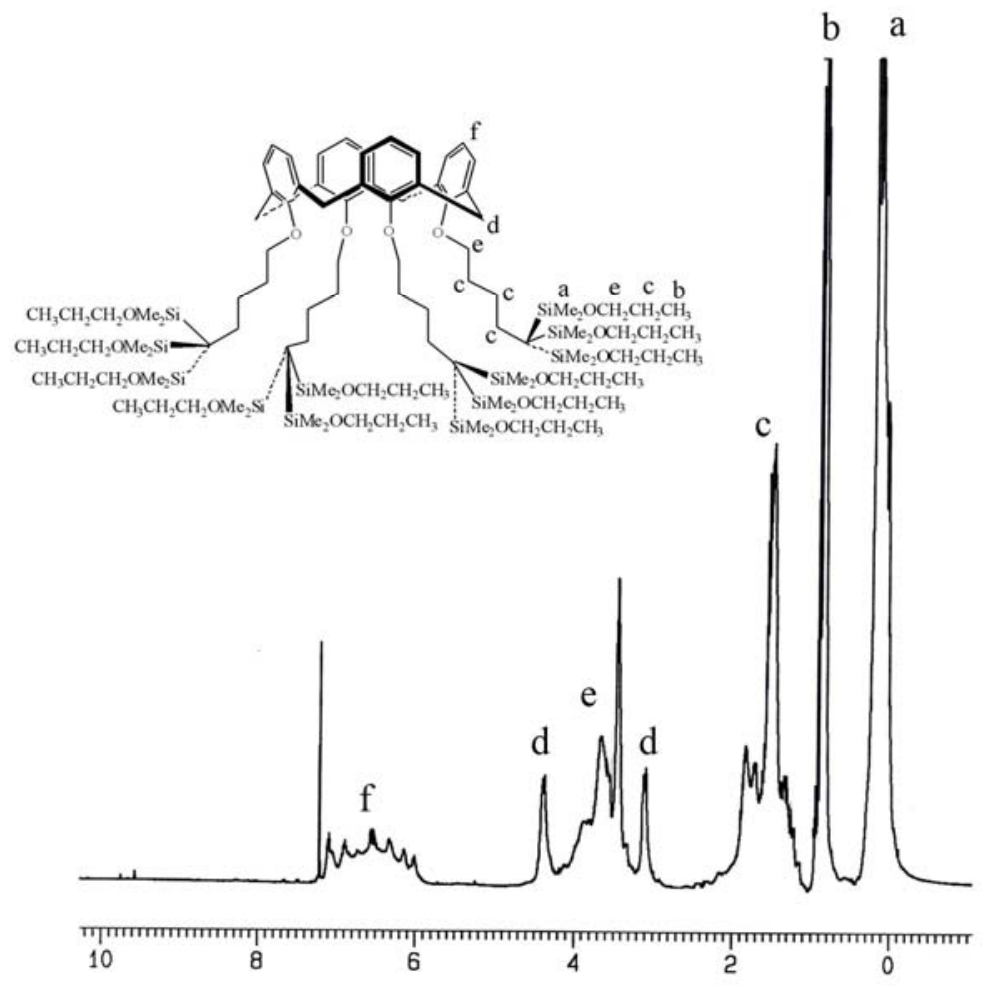

Figure 3. The ${ }^{1} \mathrm{H}$ NMR Spectrum of the calixarene 5. 


\section{Conclusions}

A series of new calixarenes bearing tris(alkoxydimethylsilyl)methyl groups were obtained in good yields from the catalytic reaction of calixarene 2 with several alcohols. Using the Speier catalyst, alcoholysis was unsuccessful under reflux conditions over seven days. However, in the presence of the Karstedt catalyst, the desired reaction went to completion in only $8 \mathrm{~h}$ at $80^{\circ} \mathrm{C}$ to afford the desired products in high yields. Primary alcohols gave higher yields than the secondary alcohols, which might be the result of the increase in steric hindrance. Furthermore, the newly-obtained calixarenes containing alkoxysilanes at the lower rim are novel calixarenes which open up a new field in the chemistry of calixarenes.

\section{Experimental Section}

General. The reactions involving organolithium reagents were carried out under dry argon. Solvents were dried by standard methods. Substrates for preparation of $\left(\mathrm{HSiMe}_{2}\right)_{3} \mathrm{CLi}$, viz. $\mathrm{HSiMe}_{2} \mathrm{Cl}, \mathrm{Mg}, \mathrm{CHBr}_{3}, n$-BuLi, lithium diisopropyl amide (LDA) and the substrate for preparation of 25,26,27,28-tetrakis[4-bromobutoxy]calix[4]arene, viz. p-tert-butylphenol, formaldehyde $35-40 \%$, NaH, DMF, 1,4-dibromobutane, and all alcohols used in the alcoholysis reactions were purchased from Merck and all alcohols were purified by standard methods. Karstedt catalyst was purchased from Aldrich. The ${ }^{1} \mathrm{H}$ NMR and ${ }^{13} \mathrm{C}$ NMR were recorded with a Bruker FT-400MHz spectrometer at room temperature and using $\mathrm{CDCl}_{3}$ as the solvent. The FTIR spectra were recorded on a Bruker- Tensor 270 spectrometer. Elemental analyses were carried out with a Heareus CHN-ORAPID instrument.

Preparation of 25,26,27,28-tetrakis(4-bromobutoxy)calix[4]arene (1). A mixture of $3.0 \mathrm{~g}$ $(7.0 \mathrm{mmol})$ of dealkylated calixarene and $2.4 \mathrm{~g}(60 \mathrm{mmol}) \mathrm{NaH}(60 \%$ in paraffin $)$ in $100 \mathrm{ml}$ of dry DMF was stirred at room temperature for $0.5 \mathrm{~h}$. Subsequently $20 \mathrm{~g}(0.1 \mathrm{mmol}) 1,4-$ dibromobutane was added. The reaction mixture was stirred at room temperature for $24 \mathrm{~h}$. Then DMF was evaporated in vacuo, and the residue was taken up in $\mathrm{CHCl}_{3}(200 \mathrm{ml})$ and washed with aqueous $1 \mathrm{~N} \mathrm{HCl}(50 \mathrm{ml} \times 2)$, brine $(50 \mathrm{ml})$ and the $\mathrm{CHCl}_{3}$ extract was dried over $\mathrm{MgSO}_{4}$. After filtration the solvent was evaporated, and the residue was subjected to column chromatography ( $n$-hexane-ethylacetate, $4: 1, R_{f}=0.80$ ), yielding $4 \mathrm{~g}(60 \%)$ a pure colourless solid. $\mathrm{Mp}=79-81^{\circ} \mathrm{C}$; ${ }^{1} \mathrm{H}$ NMR (400 MHz, $\left.\mathrm{CDCl}_{3}\right): \delta 1.96-2.15\left(\mathrm{~m}, 16 \mathrm{H}, 4 \times \mathrm{CH}_{2} \mathrm{C}_{2} \mathrm{C}_{2} \mathrm{CH}_{2}\right), 3.2(\mathrm{~d}, 4 \mathrm{H}, \mathrm{J}=13.4 \mathrm{~Hz}$, $4 \times \operatorname{ArCHAr}), 3.5\left(\mathrm{t}, 8 \mathrm{H}, \mathrm{J}=6.34 \mathrm{~Hz}, 4 \times \mathrm{CH}_{2} \mathrm{CH}_{2} \mathrm{CH}_{2} \mathrm{CH}_{2} \mathrm{O}\right), 3.9(\mathrm{t}, 8 \mathrm{H}, \mathrm{J}=6.94 \mathrm{~Hz}$, $\mathrm{CH}_{2} \mathrm{CH}_{2} \mathrm{CH}_{2} \mathrm{CH}_{2} \mathrm{Br}$ ), 4.4 (d, $4 \mathrm{H}, \mathrm{J}=13.35 \mathrm{~Hz}, 4 \times \mathrm{ArCH} \underline{\mathrm{Ar}}$ ), $6.55-6.72(\mathrm{~m}, 12 \mathrm{H}, \mathrm{Ar}) \mathrm{ppm} ;{ }^{13} \mathrm{C}$ NMR (100 MHz, $\left.\mathrm{CDCl}_{3}\right): \delta 27.94,28.54,29.85,32.70,72.94,121.24,127.27,133.85,155.09$ ppm; Anal. Calcd for $\mathrm{C}_{44} \mathrm{H}_{52} \mathrm{Br}_{4} \mathrm{O}_{4}$ (964.50): C, 54.79\%; H, 5.43\%. Found: C, 54.32\%; H, 5.15\%. Preparation of tris(dimethylsilyl)methyllithium, (HSiMe $\left.)_{3}\right)_{3} \mathrm{CLi}$, solution in $\mathrm{THF}^{19}{ }^{19}$ A $50 \mathrm{ml}$ round-bottom flask equipped with a stirrer, septum, and gas-inlet needle was charged with 
diisopropylamine $(0.53 \mathrm{~g}, 5.3 \mathrm{mmol})$ and $15 \mathrm{ml}$ of THF. The flask was placed in a water- ice bath and then $n$-Buli (3.8 ml, $1.5 \mathrm{M}$ solution in hexane) was added dropwise via syringe to form a clear yellow solution. The solution was stirred for an additional $30 \mathrm{~min}$. The lithium diisopropylamide (LDA) solution was transferred into a dropping funnel after which it was added dropwise to a 50-ml round-bottom flask containing tris(dimethylsilyl)methane, $\left(\mathrm{HSiMe}_{2}\right)_{3} \mathrm{CH},(1.0 \mathrm{~g}, 5.3 \mathrm{mmol})$, in $10 \mathrm{ml}$ THF under argon atmosphere at room temperature. The orange-red solution was stirred at ambient temperature for $10 \mathrm{~h}$.

Preparation of 25,26,27,28-tetrakis[4-(tris(dimethylsilyl)methyl)butoxy]calix[4]arene (2). To a stirred solution of $\left(\mathrm{HSiMe}_{3}\right)_{3} \mathrm{CLi}(5.3 \mathrm{mmol})$ in $\mathrm{THF}$ at $0^{\circ} \mathrm{C}$ was added $25,26,27,28$ tetrakis(4-bromobutoxy) calix[4]arene $1(1.0 \mathrm{mmol})$ in $10 \mathrm{ml} \mathrm{THF}$, and then stirred for another 2 $\mathrm{h}$ at room temperature. The reaction mixture was poured into aqueous ammonium chloride solution $(50 \mathrm{ml})$ and extracted with $\mathrm{CH}_{2} \mathrm{Cl}_{2}(2 \times 50 \mathrm{ml})$. The organic phase was washed with water $(100 \mathrm{ml})$ and dried $\left(\mathrm{Na}_{2} \mathrm{SO}_{4}\right)$, and the solvent was removed in vacuo to yield a viscous oil. A pure colourless solid $1.15 \mathrm{~g}(82 \%)$ was obtained by preparative TLC (silica gel, $n$-hexane, $\left.R_{f}=0.2\right), \mathrm{mp}=59-61^{\circ} \mathrm{C}$; FTIR $\left(\mathrm{KBr}, \mathrm{cm}^{-1}\right): 3061(\mathrm{HC}=), 2107(\mathrm{Si}-\mathrm{H}), 1589,1456(\mathrm{Ph}), 1252,896$ $\left(\mathrm{Si}-\mathrm{CH}_{3}\right) ;{ }^{1} \mathrm{H} \mathrm{NMR}\left(400 \mathrm{MHz}, \mathrm{CDCl}_{3}\right): \delta=0.1\left(\mathrm{~d}, 72 \mathrm{H},{ }^{3} \mathrm{~J}_{\mathrm{HH}}=3.74 \mathrm{~Hz}, 12 \times \mathrm{SiMe}_{2}\right), 1.51-1.58(\mathrm{~m}$, $\left.8 \mathrm{H}, \quad 4 \times \mathrm{CCH}_{2} \mathrm{CH}_{2}\right), \quad 1.66-1.71 \quad\left(\mathrm{~m}, \quad 8 \mathrm{H}, \quad 4 \quad \times \mathrm{CCH}_{2} \mathrm{CH}_{2} \mathrm{CH}_{2}\right), \quad 1.85-1.93(\mathrm{~m}, \quad 8 \mathrm{H}, \quad 4$ $\left.\times \mathrm{CCH}_{2} \mathrm{CH}_{2} \mathrm{CH}_{2} \mathrm{CH}_{2}\right), 3.1(\mathrm{~d}, 4 \mathrm{H}, \mathrm{J}=13.4 \mathrm{~Hz}, 4 \times$ ArCHAr$), 3.9(\mathrm{t}, 8 \mathrm{H}, \mathrm{J}=7.24 \mathrm{~Hz}, 4 \times$ $\left.\mathrm{CCH}_{2} \mathrm{CH}_{2} \mathrm{CH}_{2} \underline{\mathrm{CH}}_{2} \mathrm{O}\right), 3.99-4.05\left(\mathrm{~m}, 12 \mathrm{H}, 12 \times \mathrm{Si}_{\underline{H} M e}\right), 4.4(\mathrm{~d}, 4 \mathrm{H}, \mathrm{J}=13.33 \mathrm{~Hz}, 4 \times \mathrm{ArCH} \underline{\mathrm{Cr}})$, 6.56-6.62 (m, 12H, Ar) ppm; ${ }^{13} \mathrm{C} \mathrm{NMR}\left(100 \mathrm{MHz}, \mathrm{CDCl}_{3}\right): \delta=-4.06\left(\mathrm{SiMe}_{2}\right), 0.58$ $\left(\underline{\mathrm{C}}\left(\mathrm{SiMe}_{2} \mathrm{H}\right)_{3}\right), 25.09,29.51,30.29,30.69,73.70,120.90,127.18,133.81,155.64 \mathrm{ppm}$; Anal. Calcd for $\mathrm{C}_{72} \mathrm{H}_{136} \mathrm{O}_{4} \mathrm{Si}_{12}$ (1400.77): C, 61.64\%; H, 9.77\%. Found: C, 61.25\%; H, 9.63\%.

\section{General procedure for the synthesis of 25,26,27,28-tetrakis[4-(tris(alkoxydimethylsilyl) methyl)butoxy]calix[4]arene}

A $50 \mathrm{ml}$ round-bottom two-neck flask with magnetic stirring was charged with 25,26,27,28tetrakis[4-(tris(dimethylsilyl)methyl)butoxy]calix[4]arene $2(0.10 \mathrm{~g}, 0.071 \mathrm{mmol})$ and $\mathrm{ROH}$ $(20 \mathrm{ml})$ under dry argon. Karstedt catalyst $\left([\mathrm{Pt}] /[\mathrm{Si}-\mathrm{H}]=7.2 \times 10^{-3}\right)$ was then added and the reaction progress was monitored. Several samples were taken at different times and were analyzed by infrared (FTIR) spectroscopy. The mixture was stirred at $60-80^{\circ} \mathrm{C}$ until complete disappearance of the $\mathrm{Si}-\mathrm{H}$ bond in the FTIR spectra. After completion of the reaction, the mixture was allowed to cool to room temperature. Then the alcohol was evaporated under reduced pressure and the residue purified by flash column chromatography (silica gel, 10:1 $n$ hexane:ethyl acetate) to give a highly viscous oily product.

25,26,27,28-Tetrakis[4-(tris(methoxy dimethylsilyl)methyl)butoxy]calix[4]arene (3). Yield 85\%, FTIR (KBr, cm $\left.{ }^{-1}\right): 3060(\mathrm{HC}=), 2994(\mathrm{C}-\mathrm{H}), 1584,1454(\mathrm{Ph}), 1251,851$ (Si-C), 1086 (SiO), $1033(\mathrm{C}-\mathrm{O}) \mathrm{cm}^{-1} ;{ }^{1} \mathrm{H}$ NMR (400 MHz, $\mathrm{CDCl}_{3}$ ): $\delta=0.19$ (br s, 72H,24×SiMe), 1.56-1.88 (br $\left.\mathrm{m}, 24 \mathrm{H}, 4 \times \underline{\mathrm{CH}}_{2} \underline{\mathrm{CH}}_{2} \underline{\mathrm{CH}}_{2} \mathrm{CH}_{2} \mathrm{O}\right), 3.1(\mathrm{~d}, 4 \mathrm{H}, \mathrm{J}=13.17 \mathrm{~Hz}, 4 \times \mathrm{ArCHAr}$ ), 3.3 (br s, $36 \mathrm{H}$, $12 \times \mathrm{OCH}_{3}$ ), 3.4-3.9 (br m, 8H, $4 \times \mathrm{OCH}_{2} \mathrm{CH}_{2}$ ), 4.4 (d, $4 \mathrm{H}, \mathrm{J}=13.25 \mathrm{~Hz}, 4 \times \operatorname{ArCHAr}$ ), 6.55-6.80 (br m, 12H, Ar) ppm; ${ }^{13} \mathrm{C} \mathrm{NMR} \mathrm{(100} \mathrm{MHz,} \mathrm{CDCl}_{3}$ ): $\delta=-0.92$ (SiMe), 2.36, 26.1, 28.33, 30.33, 
30.77, 48.84, 73.83, 120.65, 127.06, 133.89, 155.75 ppm; Anal. Calcd for $\mathrm{C}_{84} \mathrm{H}_{160} \mathrm{O}_{16} \mathrm{Si}_{12}$ : C, 57.22; H, 9.15. Found: C,57.01; H, 8.85.

25,26,27,28-Tetrakis[4-(tris(ethoxydimethylsilyl)methyl)butoxy]calix[4]arene (4). Yield 85\%, FTIR (KBr, cm $\left.{ }^{-1}\right): 3060(\mathrm{HC}=), 2970,2898(\mathrm{C}-\mathrm{H}), 1586,1454(\mathrm{Ph}), 1252,847(\mathrm{Si}-\mathrm{C})$, $1080(\mathrm{Si}-\mathrm{O}) \mathrm{cm}^{-1} ;{ }^{1} \mathrm{H} \mathrm{NMR}\left(400 \mathrm{MHz}, \mathrm{CDCl}_{3}\right): \delta=0.18$ (br s, 72H,24×SiMe), 1.01 (br t, 36H, $\left.12 \times \underline{\mathrm{CH}_{3}} \mathrm{CH}_{2}\right), 1.59-1.95\left(\mathrm{br} \mathrm{m}, 24 \mathrm{H}, 4 \times \underline{\mathrm{CCH}}_{2} \underline{\mathrm{CH}}_{2} \underline{\mathrm{CH}}_{2} \mathrm{CH}_{2} \mathrm{O}\right), 3.1(\mathrm{~d}, 4 \mathrm{H}, \mathrm{J}=13.09 \mathrm{~Hz}$, $4 \times$ ArCHAr), 3.5-3.8 (br m, 32H, 16× $\underline{\mathrm{OCH}}_{2}$ ), 4.4 ( d, 4H, J = 13.13 Hz, 4×ArCHAr), 6.5-6.9(br m, 12H, Ar) ppm; ${ }^{13} \mathrm{C}$ NMR $\left(100 \mathrm{MHz}, \mathrm{CDCl}_{3}\right): \delta=-0.59$ (SiMe), 1.769, 22.73, 27.91, 28.68, 29.35, 30.52, 57.12, 67.14,120.84, 127.79, 131.44, 155.57 ppm; Anal. Calcd for $\mathrm{C}_{96} \mathrm{H}_{184} \mathrm{O}_{16} \mathrm{Si}_{12}$ : C, 59.70; H, 9.60. Found: C,59.45; H, 9.55.

25,26,27,28-Tetrakis[4-(tris(propoxydimethylsilyl)methyl)butoxy]calix[4]arene (5). Yield 83\%, FTIR (KBr, cm $\left.{ }^{-1}\right)$ : $3061(\mathrm{HC}=)$, 2959, $2872(\mathrm{C}-\mathrm{H}), 1586,1457(\mathrm{Ph}), 1254,847$ (Si-C), $1084(\mathrm{Si}-\mathrm{O}) \mathrm{cm}^{-1} ;{ }^{1} \mathrm{H}$ NMR $\left(400 \mathrm{MHz} \mathrm{CDCl}_{3}\right): \delta=0.19$ (br s, $\left.72 \mathrm{H}, 24 \times \mathrm{SiMe}\right), 0.9$ (br t, 36H, $12 \times \underline{\mathrm{CH}_{3}} \mathrm{CH}_{2}$ ), 1.30-1.93 (br m, 48H, $4 \times \underline{\mathrm{CCH}}_{2} \underline{\mathrm{CH}_{2}} \underline{\mathrm{CH}}_{2} \mathrm{CH}_{2} \mathrm{O}, 12 \times \mathrm{CH}_{3} \underline{\mathrm{CH}}_{2} \mathrm{CH}_{2} \mathrm{O}$ ), 3.1 (d, $4 \mathrm{H}, \mathrm{J}=$

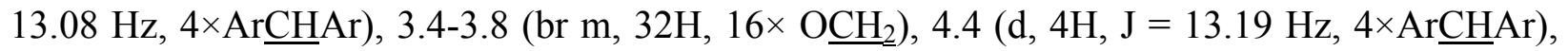
6.1-6.9 (br m, 12H, Ar) ppm; $\left.{ }^{13} \mathrm{C} \mathrm{NMR} \mathrm{(100} \mathrm{MHz,} \mathrm{CDCl}_{3}, \mathrm{ppm}\right): \delta=-0.45$ (SiMe), 2.86, 9.42, 24.8, 25.10, 29.01, 30.31, 30.70, 63.0, 73.8, 120.95, 127.70, 133.91, 155.68 ppm; Anal. Calcd for $\mathrm{C}_{108} \mathrm{H}_{208} \mathrm{O}_{16} \mathrm{Si}_{12}$ : C, 61.77; H, 9.98. Found: C, 61.45; H, 9.80 .

25,26,27,28-Tetrakis[4-(tris(butoxydimethylsilyl)methyl)butoxy]calix[4]arene (6). Yield 80\%, FTIR (KBr, cm $\left.{ }^{-1}\right)$ : $3060(\mathrm{HC}=), 2958,2868(\mathrm{C}-\mathrm{H}), 1587,1459(\mathrm{Ph}), 1251,850$ (Si-C), $1091(\mathrm{Si}-\mathrm{O}) \mathrm{cm}^{-1} ;{ }^{1} \mathrm{H} \mathrm{NMR}\left(400 \mathrm{MHz}, \mathrm{CDCl}_{3}\right.$ ): $\delta=0.18$ (br s, 72H,24×SiMe), 0.9 (br t, 36H, $12 \times \underline{\mathrm{CH}_{3}} \mathrm{CH}_{2}$ ), 1.2-1.9 (br m, 72H, $4 \times \underline{\mathrm{CCH}}_{2} \underline{\mathrm{CH}_{2}} \underline{\mathrm{CH}_{2}} \underline{\mathrm{CH}}_{2} \mathrm{O}, 12 \times \mathrm{CH}_{3} \underline{\mathrm{CH}}_{2} \underline{\mathrm{CH}}_{2} \mathrm{CH}_{2} \mathrm{O}$ ), 3.1 (d, $4 \mathrm{H}, \mathrm{J}$ $=13.08 \mathrm{~Hz}, 4 \times \mathrm{Ar} \underline{\mathrm{CHAr}}), 3.3-3.9$ (br m, 32H, $\left.16 \times \mathrm{OCH}_{2}\right), 4.4(\mathrm{~d}, 4 \mathrm{H}, \mathrm{J}=13.10 \mathrm{~Hz}$, $4 \times$ ArCHAr), 6.3-6.9(br m, 12H, Ar) ppm; $\left.{ }^{13} \mathrm{C} \mathrm{NMR} \mathrm{(100} \mathrm{MHz,} \mathrm{CDCl}_{3}, \mathrm{ppm}\right): \delta=-0.01$ (SiMe), 9.1, 12.9, 18.1, 24.9, 29.10, 30.29, 30.40, 33.5, 60.9, 73.7, 119.8, 126.5, 132.7, 155.3 ppm; Anal. Calcd for $\mathrm{C}_{120} \mathrm{H}_{232} \mathrm{O}_{16} \mathrm{Si}_{12}$ : C, 63.54; H, 10.31. Found: C, 63.40; H, 10.21.

25,26,27,28-tetrakis[4-(tris(pentoxydimethylsilyl)methyl)butoxy]calix[4]arene (7). Yield 80\%, FTIR (KBr, cm $\left.{ }^{-1}\right): 3061(\mathrm{HC}=), 2953,2867(\mathrm{C}-\mathrm{H}), 1513,1459(\mathrm{Ph}), 1252,840(\mathrm{Si}-\mathrm{C})$, $1092(\mathrm{Si}-\mathrm{O}) \mathrm{cm}^{-1} ;{ }^{1} \mathrm{H}$ NMR $\left(400 \mathrm{MHz}, \mathrm{CDCl}_{3}\right.$ ): $\delta=0.18$ (br s, 72H,24×SiMe), 0.9 (br t, 36H, $12 \times \underline{\mathrm{CH}_{3}} \mathrm{CH}_{2}$ ), 1.3-1.9 (br m, $96 \mathrm{H}, 4 \times \underline{\mathrm{CCH}}_{2} \underline{\mathrm{CH}}_{2} \underline{\mathrm{CH}}_{2} \mathrm{CH}_{2} \mathrm{O}, 12 \times \mathrm{CH}_{3} \underline{\mathrm{CH}}_{2} \underline{\mathrm{CH}_{2}} \underline{\mathrm{CH}_{2}} \mathrm{CH}_{2} \mathrm{O}$ ), 3.1 (d, $4 \mathrm{H}, \mathrm{J}=12.09 \mathrm{~Hz}, 4 \times \mathrm{Ar} \underline{\mathrm{CH}} \mathrm{Ar}), 3.4-3.9$ (br m, 32H, 16× $\left.\underline{\mathrm{OCH}}_{2}\right), 4.4(\mathrm{~d}, 4 \mathrm{H}, \mathrm{J}=11.5 \mathrm{~Hz}$, $4 \times$ ArCHAr), $6.3-7.0$ (br m, 12H, Ar) ppm; $\left.{ }^{13} \mathrm{C} \mathrm{NMR} \mathrm{(100} \mathrm{MHz,} \mathrm{CDCl}_{3}\right): \delta=0.0(\mathrm{SiMe}), 9.5$, 13.1, 21.5, 24.7, 27.1, 29.1, 30.01, 30.4, 31.3, 61.08, 73.5, 119.4, 127.7, 132.3, 154.2 ppm; Anal. Calcd for $\mathrm{C}_{132} \mathrm{H}_{256} \mathrm{O}_{16} \mathrm{Si}_{12}$ : C, 65.07; H, 10.59. Found: C, 64.70; H, 10.24.

25,26,27,28-Tetrakis[4-(tris(hexoxydimethylsilyl)methyl)butoxy]calix[4]arene (8). Yield 80\%, FTIR (KBr, cm $\left.{ }^{-1}\right)$ : $3060(\mathrm{HC}=), 2930,2864(\mathrm{C}-\mathrm{H}), 1582,1459(\mathrm{Ph}), 1251,856(\mathrm{Si}-\mathrm{C})$, $1094(\mathrm{Si}-\mathrm{O}) \mathrm{cm}^{-1} ;{ }^{1} \mathrm{H} \mathrm{NMR}\left(400 \mathrm{MHz}, \mathrm{CDCl}_{3}\right.$ ): $\delta=0.19$ (br s, 72H,24×SiMe), 0.89 (br t, 36H, $12 \times \underline{\mathrm{CH}_{3}} \mathrm{CH}_{2}$ ), 1.3-1.9 (br m, $120 \mathrm{H}, 4 \times \underline{\mathrm{CCH}}_{2} \underline{\mathrm{CH}_{2}} \underline{\mathrm{CH}_{2}} \underline{\mathrm{CH}}_{2} \mathrm{O}, 12 \times \mathrm{CH}_{3} \underline{\mathrm{CH}}_{2} \underline{\mathrm{CH}_{2}} \underline{\mathrm{CH}_{2}} \underline{\mathrm{CH}_{2}} \underline{\mathrm{CH}}{ }_{2} \mathrm{O}$ ), 3.1 (d, $4 \mathrm{H}, \mathrm{J}=11.42 \mathrm{~Hz}, 4 \times \operatorname{ArCHAr}$ ), 3.4-3.9 (br m, 32H, 16× $\mathrm{OCH}_{2}$ ), 4.4 ( d, $4 \mathrm{H}, \mathrm{J}=12.9 \mathrm{~Hz}$, $4 \times$ ArCHAr), $6.3-7.0$ (br m, $12 \mathrm{H}, \mathrm{Ar}) \mathrm{ppm} ;{ }^{13} \mathrm{C} \mathrm{NMR}\left(100 \mathrm{MHz}, \mathrm{CDCl}_{3}, \mathrm{ppm}\right): \delta=0.1$ (SiMe), 
9.6, 12.9, 21.5, 24.02, 25.01, 29.30, 30.21, 30.50, 30.7, 31.7, 61.3, 73.05, 120.50, 126.83, 133.3, 155.07 ppm; Anal. Calcd for $\mathrm{C}_{144} \mathrm{H}_{280} \mathrm{O}_{16} \mathrm{Si}_{12}: \mathrm{C}, 66.40 ; \mathrm{H}, 10.83$. Found: C, 66.15; H, 10.60 .

25,26,27,28-Tetrakis[4-(tris(1-methylethoxydimethylsilyl)methyl)butoxy]calix[4]arene (9). Yield 77\%, FTIR (KBr, cm $\left.{ }^{-1}\right): 3063(\mathrm{HC}=), 2964,2866(\mathrm{C}-\mathrm{H}), 1610,1497(\mathrm{Ph}), 1254,839(\mathrm{Si}-$ C), $1061(\mathrm{Si}-\mathrm{O}) \mathrm{cm}^{-1} ;{ }^{1} \mathrm{H}$ NMR (400 MHz, $\mathrm{CDCl}_{3}$ ): $\delta=0.19$ (br s, 72H,24×SiMe), 1.17 (br d, $\left.72 \mathrm{H}, 12 \times\left(\underline{\mathrm{CH}}_{3}\right)_{2} \mathrm{CH}\right), 1.7-2.2$ (br m, $\left.24 \mathrm{H}, 4 \times \mathrm{CCH}_{2} \underline{\mathrm{CH}}_{2} \mathrm{CH}_{2} \mathrm{CH}_{2} \mathrm{O}\right), 3.1(\mathrm{~d}, 4 \mathrm{H}, \mathrm{J}=13.32 \mathrm{~Hz}$, $4 \times$ ArCHAr), 3.6-4.0 (br m, $\left.20 \mathrm{H}, 4 \times \mathrm{OCH}_{2}, 12 \times\left(\mathrm{CH}_{3}\right)_{2} \underline{\mathrm{CH}}\right), 4.4(\mathrm{~d}, 4 \mathrm{H}, \mathrm{J}=13.30 \mathrm{~Hz}$, $4 \times \mathrm{ArCH} A r), 6.3-3.9$ (br m, $12 \mathrm{H}, \mathrm{Ar}$ ) ppm; ${ }^{13} \mathrm{C}$ NMR (100 MHz, $\left.\mathrm{CDCl}_{3}, \mathrm{ppm}\right): \delta=0.3(\mathrm{SiMe})$, 10.05, 24.7, 25.0, 29.1, 29.9, 30.3, 63.0, 73.1, 120.21, 126.51, 133.4, 155.54 ppm; Anal. Calcd for $\mathrm{C}_{108} \mathrm{H}_{208} \mathrm{O}_{16} \mathrm{Si}_{12}$ : C, 61.77; H, 9.98. Found: C, 61.44; H, 9.65.

\section{5,26,27,28-Tetrakis[4-(tris(1-methylpropoxydimethylsilyl)methyl)butoxy]calix[4]arene}

(10). Yield 75\%, FTIR (KBr, cm $\left.{ }^{-1}\right): 3061$ (HC=), 2969, $2877(\mathrm{C}-\mathrm{H}), 1541,1457$ (Ph), 1253, 842 (Si-C), 1108 (Si-O) cm ${ }^{-1} ;{ }^{1} \mathrm{H}$ NMR (400 MHz, $\mathrm{CDCl}_{3}$ ): $\delta=0.19$ (br s, 72H, 24×SiMe), 0.86 (br t, $\left.36 \mathrm{H}, \quad 12 \times \mathrm{CH}_{3} \mathrm{CH}_{2}\right), \quad 1.1 \quad$ (br d, $\left.36 \mathrm{H}, \quad 12 \times\left(\underline{\mathrm{CH}}_{3}\right) \mathrm{OCHCH} 2\right), \quad 1.4-1.9(\mathrm{br} \quad \mathrm{m}, \quad 48 \mathrm{H}$, $\left.4 \times \mathrm{CCH}_{2} \underline{\mathrm{CH}}_{2} \underline{\mathrm{CH}}_{2} \mathrm{CH}_{2} \mathrm{O}, 12 \times\left(\mathrm{CH}_{3}\right) \mathrm{OCHCH}_{2} \mathrm{CH}_{3}\right), 3.1$ (d, $\left.4 \mathrm{H}, \mathrm{J}=13.13 \mathrm{~Hz}, 4 \times \mathrm{ArCHAr}\right), 3.6-4.0$ (br m, 20H, $\left.4 \times \mathrm{OCH}_{2}, 12 \times\left(\mathrm{CH}_{3}\right) \mathrm{OCHCH}_{2}\right), 4.4(\mathrm{~d}, 4 \mathrm{H}, \mathrm{J}=13.0 \mathrm{~Hz}, 4 \times \mathrm{ArCHAr}), 6.3-6.9$ (br m, $12 \mathrm{H}, \mathrm{Ar}) \mathrm{ppm} ;{ }^{13} \mathrm{C} \mathrm{NMR}\left(100 \mathrm{MHz}, \mathrm{CDCl}_{3}, \mathrm{ppm}\right): \delta=0.4$ (SiMe), 9.90, 14.2, 21.5, 24.42, 29.01, 29.95, 30.40, 31.12, 68.4, 72.29, 119.70, 126.14, 132.7, $154.56 \mathrm{ppm}$; Anal. Calcd for $\mathrm{C}_{120} \mathrm{H}_{232} \mathrm{O}_{16} \mathrm{Si}_{12}$ : C, 63.54; H, 10.31. Found: C, 63.40; H, 10.26 .

\section{5,26,27,28-Tetrakis[4-(tris(2-methylpropoxydimethylsilyl)methyl)butoxy]calix[4]arene}

(11). Yield 75\%, FTIR (KBr, cm $\left.{ }^{-1}\right): 3063(\mathrm{HC}=)$, 2959, 2877 (C-H), 1548, $1462(\mathrm{Ph}), 1253,849$ (Si-C), 1085 (Si-O) cm ${ }^{-1} ;{ }^{1} \mathrm{H}$ NMR (400 MHz, $\mathrm{CDCl}_{3}$ ): $\delta=0.20$ (br s, 72H,24×SiMe), 0.88 (br d, $\left.72 \mathrm{H}, 12 \times\left(\underline{\mathrm{CH}_{3}}\right)_{2} \mathrm{CH}\right), 1.3-1.9\left(\mathrm{br} \mathrm{m}, 36 \mathrm{H}, 4 \times \mathrm{C \underline {CH }}_{2} \underline{\mathrm{CH}_{2}} \underline{\mathrm{CH}_{2}} \mathrm{CH}_{2} \mathrm{O}, 12 \times\left(\mathrm{CH}_{3}\right)_{2} \underline{\mathrm{CHCH}_{2}}\right), 3.1(\mathrm{~d}, 4 \mathrm{H}$, $\mathrm{J}=10.61 \mathrm{~Hz}, 4 \times \operatorname{Ar} \underline{\mathrm{CHAr}}), 3.3-3.8\left(\mathrm{br} \mathrm{m}, 32 \mathrm{H}, 16 \times \mathrm{OCH}_{2}\right), 4.4(\mathrm{~d}, 4 \mathrm{H}, \mathrm{J}=9.32 \mathrm{~Hz}, 4 \times \operatorname{ArCHAr})$, 6.3-6.9(br m, $12 \mathrm{H}, \mathrm{Ar}) \mathrm{ppm} ;{ }^{13} \mathrm{C}$ NMR $\left(100 \mathrm{MHz}, \mathrm{CDCl}_{3}, \mathrm{ppm}\right): \delta=0.001$ (SiMe), 8.5, 17.8, 24.57, 28.9, 29.68, 30.29, 30.6, 67.9, 73.4, 120.84, 126.7, 133.48, 155.5 ppm; Anal. Calcd for $\mathrm{C}_{120} \mathrm{H}_{232} \mathrm{O}_{16} \mathrm{Si}_{12}$ : C, 63.54; H, 10.31. Found: C, 63.25; H, 10.10 .

25,26,27,28-Tetrakis[4-(tris(2-chloroethoxydimethylsilyl)methyl)butoxy]calix[4]arene (12). Yield 85\%, FTIR (KBr, cm $\left.{ }^{-1}\right)$ : $3062(\mathrm{HC}=), 2956,2867(\mathrm{C}-\mathrm{H}), 1585,1457(\mathrm{Ph}), 1253,850$ (SiC), 1107 (Si-O) $\mathrm{cm}^{-1} ;{ }^{1} \mathrm{H}$ NMR (400 MHz, $\mathrm{CDCl}_{3}$ ): $\delta=0.19$ (br s, $\left.72 \mathrm{H}, 24 \times \mathrm{SiMe}\right), 1.5-1.8(\mathrm{br} \mathrm{m}$, $24 \mathrm{H}, 4 \times \underline{\mathrm{CCH}}_{2} \underline{\mathrm{CH}}_{2} \underline{\mathrm{CH}}_{2} \mathrm{CH}_{2} \mathrm{O}$ ), 3.1 (d, $4 \mathrm{H}, \mathrm{J}=13.44 \mathrm{~Hz}, 4 \times \mathrm{ArCHAr}$ ), 3.4-3.9 (br m, $56 \mathrm{H}, 16 \times$ $\left.\mathrm{OCH}_{2}, 12 \times \underline{\mathrm{CH}}_{2} \underline{\mathrm{Cl}}\right), 4.3(\mathrm{~d}, 4 \mathrm{H}, \mathrm{J}=14.33 \mathrm{~Hz}, 4 \times \mathrm{ArCHAr}$ ), 6.3-7.0(br m, $12 \mathrm{H}, \mathrm{Ar}) \mathrm{ppm} ;{ }^{13} \mathrm{C}$ NMR (100 MHz, $\left.\mathrm{CDCl}_{3}, \mathrm{ppm}\right): \delta=-0.2$ (SiMe), 8.8, 26.91, 28.68, 29.35, 30.52, 44.10, 57.12, 67.14,120.84, 127.79, 131.44, 155.57 ppm; Anal. Calcd for $\mathrm{C}_{96} \mathrm{H}_{172} \mathrm{Cl}_{12} \mathrm{O}_{16} \mathrm{Si}_{12}: \mathrm{C}, 49.17 ; \mathrm{H}$, 7.39. Found: $\mathrm{C}, 48.90 ; \mathrm{H}, 8.20$. 


\section{References}

1. (a) Gutsche, C. D. Calixarenes; The Royal Society of Chemistry: London, 1989. (b) Bohmer, V. Angew. Chem., Int. Ed. 1995, 34, 713. (c) Gutsche, C. D. Calixarenes Revisited; The Royal Society of Chemistry: London, 1998. (d) Mondolini, L.; Ungaro, R., Eds. Calixarenes in Action; Imperial College Press: London, 2000. (e) Asfari, Z.; Bohmer, V.; Harrowfield, J.; Vicens, J., Eds. Calixarenes 2001; Kluwer Academic Publishers: Dordrecht, 2001.

2. Beer, P. D.; Gale, P. A. Angew. Chem., Int. Ed. 2001, 40, 486.

3. Matthews, S. E.; Beer, P. D. Calixarenes 2001; Kluwer Academic Publishers: Dordrecht, 2001, 421.

4. Hudrlik, P. F.; Hudrlik, A. M.; Arasho, J. Abstracts, $60^{\text {th }}$ Southwest Regional Meeting of the American Chemical Society, Fort Worth. TX. Sept 29-Oct 5, 2004.

5. (a) Chuit, C.; Corriu, R. J. P.; Reye, C.; Young, J. C. Chem. Rev. 1993, 93, 1371. (b) Chuit, C.; Corriu, R. J. P.; Reye, C. In Chemistry of Hypervalent Comounds; Akiba, K., Ed.; WileyVCH: New York, 1999; pp 81-146. (c) Kira, M.; Zhang, L. C. In Chemistry of Hypervalent Compounds; Akiba, K., Ed.; Wiley-VCH: New York, 1999; pp 147-169.

6. Ihm, H.; Paek, K. Bull. Korean Chem. Soc. 1995, 16, 71.

7. Billo, F.; Musau, R. M.; Whiting, A. Arkivoc 2006, (x), 199.

8. Safa, K. D.; Oskoei, Y. M. J. Organomet. Chem. 2009, doi:10.1016/j.jorganchem. 2009.11.029

9. Hudrlik, P. F; Hudrlik, A. M. J. Org. Chem. 2007, 72, 7558.

10. Hudrlik, P. F; Wondwossen, D. J. Org. Chem. 2007,72, 8107.

11. Blanda, M. T.; Frels, J. Supramolecular Chemistry 1998, 9, 255.

12. Kocienski, P. J. Protecting Groups; Thieme: Stuttgart, 1994, pp 28.

13. Mrsny, R. J. Site-Specefic Drug Delivery in the Gastrointestinal Tract, In Controlled Drug Delivery 1997; Chap.6, p 107.

14. Schubert, U.; Lorenz, C. Chem Ber. 1995, 128, 1267.

15. Brook, M. A. Silicon In Organic, Organometalic and Polymer Chemistry, WileyInterscience: New York, 2003.

16. Safa, K. D.; Tofangdarzadeh, Sh.; Heydari, H. Hetroatom Chemistry 2008, 19, 365.

17. Eaborn, C.; Hitchcok, P. B. J. Organomet. Chem. 1983, 252, 281.

18. Eric, J.; Hawrelak, F.; Ladipo, T. Organometallics 1999, 18, 1804.

19. Safa, K. D.; Shahrivar, M.; Tofangdarzadeh, S.; Hassanpour, A. Tetrahedron 2007, 63, 3189. 